\title{
WR-3 Waveguide Bandpass Filters Fabricated Using High Precision CNC Machining and SU-8 Photoresist Technology
}

\author{
Hao Yang ${ }^{(0)}$, Yuvaraj Dhayalan, Xiaobang Shang ${ }^{\left({ }^{\circ}\right.}$, Member, IEEE, Michael J. Lancaster, Senior Member, IEEE, \\ Bo Liu ${ }^{-}$, Senior Member, IEEE, Hui Wang, Manju Henry, and Peter G. Huggard, Senior Member, IEEE
}

\begin{abstract}
This paper presents two WR-3 band (220-325 GHz) filters, one fabricated in metal using high precision computer numerically controlled milling and the other made with metallized SU-8 photoresist technology. Both are based on three coupled resonators, and are designed for a $287.3-295.9-\mathrm{GHz}$ passband, and a 30-dB rejection between 317.7 and 325.9 GHz. The first filter is an extracted pole filter coupled by irises, and is precision milled using the split-block approach. The second filter is composed of three silver-coated SU-8 layers, each $432 \mu$ m thick. The filter structures are specially chosen to take advantage of the fabrication processes. When fabrication tolerances are accounted for, very good agreement between measurements and simulations are obtained, with median passband insertion losses of 0.41 and $0.45 \mathrm{~dB}$ for the metal and SU-8 devices, respectively. These two filters are potential replacements of frequency selective surface filters used in heterodyne radiometers for unwanted sideband rejection.
\end{abstract}

Index Terms-Micromachining, SU-8, terahertz components, waveguide filter.

\section{INTRODUCTION}

$\mathbf{M}$ ULTICHANNEL air and spaceborne sounders are employed for spectroscopic characterization of the Earth's atmosphere [1]. These instruments perform molecular spectroscopy at millimeter and submillimeter wavelengths in relatively narrow frequency channels. Within the instrument, linearly polarized signals are frequency demultiplexed by a

Manuscript received September 19, 2017; accepted November 6, 2017. Date of publication December 7, 2017; date of current version January 9, 2018. This work was supported by the U.K. Engineering and Physical Science Research Council under Contract EP/M016269/1. (Corresponding author: Hao Yang.)

H. Yang, Y. Dhayalan, and M. J. Lancaster are with the Department of Electronic, Electrical and Systems Engineering, University of Birmingham, Birmingham B15 2TT, U.K. (e-mail: hxy297@bham.ac.uk; yuvaraj@gmail.com; m.j.lancaster@bham.ac.uk).

X. Shang was with the Department of Electronic, Electrical and Systems Engineering, University of Birmingham, Birmingham B15 2TT, U.K. He is now with the National Physical Laboratory, Teddington TW11 OLW, U.K. (e-mail: xiaobang.shang@npl.co.uk).

B. Liu is with the School of Electrical, Electronic, and System Engineering, University of Birmingham, Birmingham B15 2TT, U.K., and also with the Department of Computing, Wrexham Glyndwr University, Wrexham LL11 2AW, U.K. (e-mail: liubo168@gmail.com).

H. Wang, M. Henry, and P. G. Huggard are with the Space Science and Technology Department, Rutherford Appleton Laboratory, Oxfordshire OX110QX, U.K. (e-mail: hui.wang@stfc.ac.uk; manju.henry@stfc.ac.uk; peter.huggard@stfc.ac.uk).

Color versions of one or more of the figures in this paper are available online at http://ieeexplore.ieee.org.

Digital Object Identifier 10.1109/TTHZ.2017.2775441 quasi-optical feed chain using frequency selective surfaces (FSSs). FSSs are also used to prevent the signals in the unwanted sideband from reaching the double sideband heterodyne mixers [2]. As the atmospheric signals are weak, the sideband rejecting FSS needs to have a very low insertion loss and a high isolation between two adjacent channels which are close to each other in frequency [3]. For instance, the FSS reported in [3] transmits $316.5-325.5-\mathrm{GHz}$ radiation with a maximum insertion loss of $0.6 \mathrm{~dB}$ and achieves greater than $30-\mathrm{dB}$ rejection from 349.5 to $358.5 \mathrm{GHz}$. To achieve this specification, a transmission zero is required and we believe this paper describes the first filter to achieve this at these high frequencies.

Waveguide technology is potentially an attractive alternative to FSS for sideband selection, due to its low loss and the possibility to construct lower volume filters. For waveguide components in general, device dimensions decrease as frequency increases. This means a reduction in size and mass of components, but also a tighter dimensional tolerance is required during fabrication. Different micromachining techniques, such as computer numerically controlled (CNC) milling [4]-[6], Si deep reactive ion etching (DRIE) [7]-[9], lithographic micromachining technique [10], and SU-8 photoresist technology [11]-[13], have been developed and employed to achieve high-dimensional accuracy in the fabrication of high-frequency waveguide filters. Laser micromachining [14] and 3-D printing [14] have also been utilized for high-frequency filters by the authors using different designs at about $100 \mathrm{GHz}$. This paper is complementary showing how alternative technologies cope, at higher frequencies, for a filter with demanding specifications.

CNC milling is a traditional way of fabricating metal waveguide components, especially at lower frequencies. Waveguide components fabricated by $\mathrm{CNC}$ milling with excellent performance have been reported. Authors in [4] and [5] describe fourth-order W-band filters. In [4], the filter is measured to have $0.5-\mathrm{dB}$ insertion loss and a $4.53 \%(4.20 \mathrm{GHz})$ bandwidth, and in [5], the filter is measured to have 0.6-dB insertion loss and a $10 \%(10 \mathrm{GHz})$ bandwidth. For the 220-325-GHz WR-3 band, waveguide features and tolerances decrease by a factor of around 3. Fabricating waveguide filters at such a frequency requires an expensive, high precision $\mathrm{CNC}$ mill. Filter design flexibility is also limited by tool sizes and depth to diameter aspect ratios [5]. However, the limits of conventional CNC milling are still being pushed: examples of WR-3 band CNC milled filter 
can be found in [6], where two fourth-order bandpass filters are presented, one with measured 0.7-dB insertion loss and $8.77 \%$ $(22.6 \mathrm{GHz})$ bandwidth and another with measured $0.5-\mathrm{dB}$ insertion loss and $9.83 \%(25.2 \mathrm{GHz})$ bandwidth. In this paper, we present a WR-3 band CNC milled extracted pole filter with an improved performance, which is designed specifically for sideband rejection.

SU-8 photoresist is a promising technology for manufacturing millimeter and submillimeter waveguide components, and is used for the second filter discussed in this paper. SU-8 is a photolithographically patterned, epoxy-based, resin that is resistant to organic solvents once cured, and can have a thickness ranging from $0.5 \mu \mathrm{m}$ to $1 \mathrm{~mm}$ [15]. There are several advantages of using SU-8 micromachining over CNC machining. For example, SU-8 can achieve a similar high-dimensional accuracy with potentially lower cost. It may also allow sharper internal corners and higher corner radius to depth ratios. Meanwhile, the SU-8 process is a batch fabrication which allows repeatability between devices as well as production of several devices in a single fabrication run [15]. Compared with DRIE, standard photolithography processes are used with SU-8 with better surface roughness on the sidewalls of waveguide structures [15]. SU-8 photoresist technology has been employed to demonstrate filters in the WR-10 [11], WR-3 [12], and WR-1.5 bands [13]. In this paper, a new WR-3 band waveguide filter is designed for the SU-8 process to meet a specification similar to the FSS filter described above.

For both the CNC and the SU-8 filters presented and compared below, the specifications in [3] were adjusted to cope with the available measurement capability. Both filters have transmission zeros with the $\mathrm{CNC}$ filter having a conventional design; however, the SU8 filter is a completely new design topology to produce the transmission zero. Frequencies have been scaled down by a factor of 1.1 , so that the new passband is $287.3-295.9 \mathrm{GHz}$ and the stopband attenuation specification becomes $30 \mathrm{~dB}$ between 317.7 and $325.9 \mathrm{GHz}$.

Structural details and design methods of the two filters are presented in Sections II and III, which is followed by a description of the fabrication process in Section IV. Measurements and discussions are presented in Section V, and conclusions are given in Section VI.

\section{Design of CNC Milled Extracted Pole Filter}

The CNC filter design is shown in Fig. 1. It is based on three coupled resonators operating at $\mathrm{TE}_{101}$ mode and an extracted pole resonator [16]. The material conductivity is assumed to be that of gold (i.e., $4.10 \times 10^{7} \mathrm{~S} / \mathrm{m}$ ). A third-order waveguide cavity filter which has a Chebyshev response was designed first, using the synthesis technique described in [17], to have a center frequency of $291.6 \mathrm{GHz}$, a bandwidth of $3 \%(8.6 \mathrm{GHz})$, and a return loss in the passband of $\geq 20 \mathrm{~dB}$. For this structure, an iris between the test ports and the first/last resonators controls the external coupling $\left(Q_{e}\right)$; the iris between resonators 1 and 2 (or resonators 2 and 3) controls the coupling coefficient $k_{12}$ (or $\left.k_{23}\right)$. To meet this specification, the external $Q$ and coupling coefficients are calculated to be $Q_{e 1}=Q_{e 3}=28.87$,

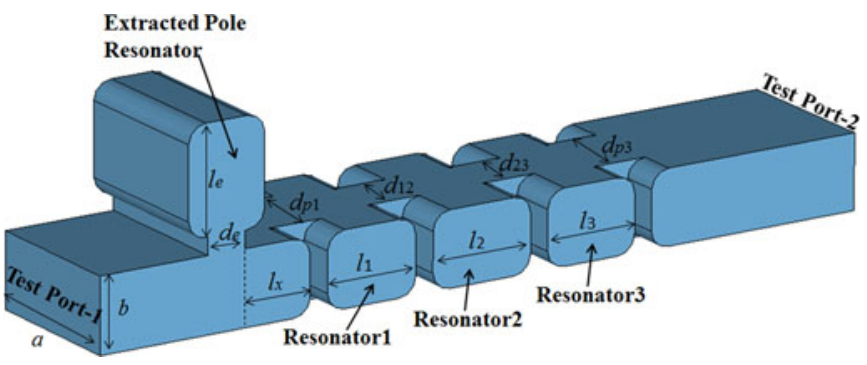

Fig. 1. Diagram of extracted pole filter structure. $a=864, b=432,1_{1}=$ $l_{3}=510, l_{2}=517, l_{e}=619, l_{x}=383, d_{e}=205, d_{p 1}=d_{p 3}=432$, and $d_{12}=d_{23}=309$ (unit: $\mu \mathrm{m}$ ).

$k_{12}=k_{23}=0.030$. To meet the upper stopband specification, a steep roll-off is needed on the high frequency side of the passband. It has been shown [16] that the selectivity of a conventional waveguide filter can be improved by using inductively coupled stopband cavities connected to the broad wall of the waveguide [18]. In [19], the advantages of this extracted pole technique have been demonstrated. A single extracted pole resonator, added to the third-order waveguide filter using the method of authors in [20] and [21], provides a transmission zero in the rejection band and achieves a very high cut-off rate into the upper sideband.

In order to be compatible with $\mathrm{CNC}$ milling process, the corners of the resonators have a radius of $0.10 \mathrm{~mm}$ (see Fig. 1) to permit fabrication with a $0.20-\mathrm{mm}$ end mill. Meanwhile, the minimum dimension in the $E$-plane must also be larger than $0.20 \mathrm{~mm}$. After initial design of the structure using the coupling matrix approach [17], full-wave simulation and optimization for this filter are carried out by CST Microwave Studio (version 2016) using the Trust Region Framework algorithm. The cavity dimensions achieved after optimization are shown in Fig. 1. To give an idea of scale, the total length of the filter structure is $4 \mathrm{~mm}$.

In order to test the filter, a CNC machined block which contains the filter is designed. The input and output waveguides have both been extended by $8 \mathrm{~mm}$ in order to accommodate standard waveguide flanges and screws. A 20-mm length of straight WR-3 waveguide is included as a measurement reference in the same block as the waveguide filter. Standard UG387 waveguide flanges were machined in the block. By utilizing an $E$-plane split-block technique, the transmission loss is minimized as no surface current flows across the contact plane. The simulation results after optimization for $S$-parameters are shown in Fig. 2. The predicted passband insertion loss for the extracted pole filter (4 $\mathrm{mm}$ filter structure) is below $0.4 \mathrm{~dB}$ and the rejection in the unwanted sideband is above $30 \mathrm{~dB}$. Passband reflectivity, $S_{11}$, is below $-20 \mathrm{~dB}$.

\section{Design OF SU-8 Micromachined FiLTER}

The SU-8 photoresist filter is designed [16] to meet the same specifications. It exploits the stacked metalized layer approach associated with SU-8 [22]. The filter has three coupled resonators and a cross-coupling between the first and third resonators and is shown in Fig. 3. With this topology and by setting the frequency of the transmission zero at 


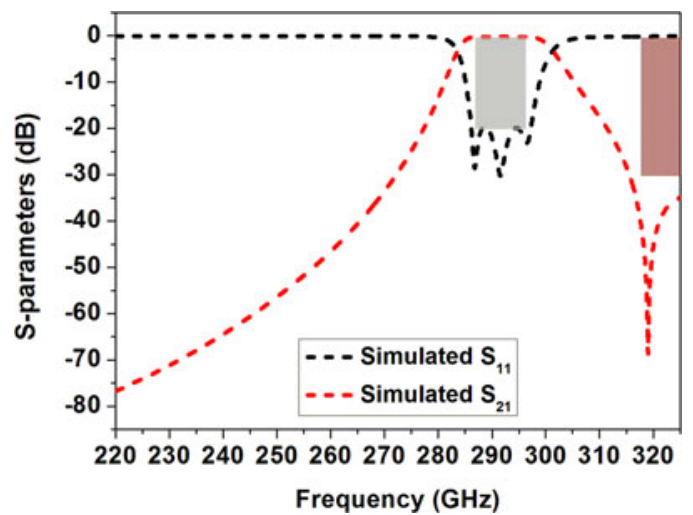

Fig. 2. Simulated $S$-parameters for the CNC milled extracted pole filter. Passband and stopband specifications are shown in gray and red, respectively.

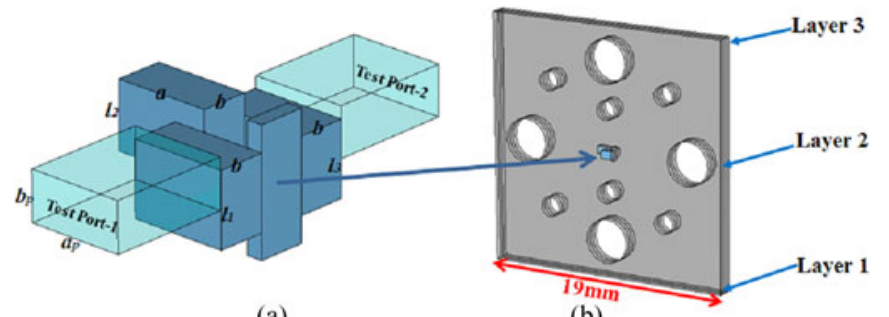

(a)

(b)

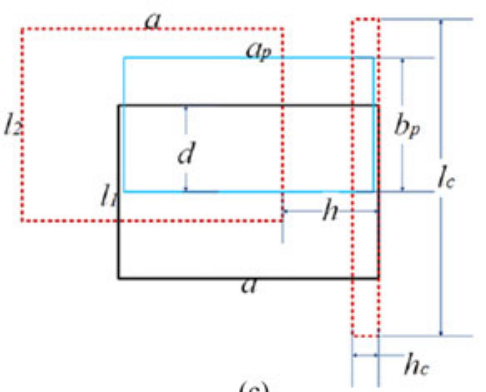

(c)

Fig. 3. Diagram of WR-3 band filter formed of three SU-8 layers with the same thickness of $432 \mu \mathrm{m}$. (a) Diagram of the filter structure. Dimensions of the test ports are: $a_{p}=864 \mu \mathrm{m}, b_{p}=432 \mu \mathrm{m}$. Dimensions of the resonators are: $a=876 \mu \mathrm{m}, b=432 \mu \mathrm{m}, l_{1}=l_{3}=647 \mu \mathrm{m}$, and $l_{2}=589 \mu \mathrm{m}$. (b) Illustration of the whole filter device including dowel holes and screw clearance holes designed to match the UG-387 waveguide flange. (c) Perspective front view of the filter structure. The blue rectangle represents the input/output of test port. The black rectangle represents the first (or third) resonator, whereas the cavities in the second layer are represented by red rectangles. $d=327 \mu \mathrm{m}$, $h=578 \mu \mathrm{m}, l_{c}=1100 \mu \mathrm{m}$, and $h_{c}=143 \mu \mathrm{m}$.

$317.7 \mathrm{GHz}$, which is the lower boundary of the upper stopband, external $Q$ and coupling coefficients are calculated as: $Q_{e 1}=Q_{e 3}=27.65, k_{12}=k_{23}=0.031, k_{13}=0.006, k_{11}=$ $k_{33}=0.002, k_{22}=-0.006$. Each of the silver-coated SU-8 layers has a thickness of $432 \mu \mathrm{m}$ and contains one resonator. As shown in Fig. 3(a), the value of the SU-8 layer thickness determines the WR-3 waveguide resonator height, $b$. Since the central resonator couplings $k_{12}$ and $k_{23}$ are equal, layers 1 and 3 are identical and the whole structure is symmetrical. Rather than controlling the coupling through a conventional iris, the relative positions of the resonators are shifted to obtain the desired coupling coefficients. In other words, the horizontal displacement $h$, shown in Fig. 3(c), determinates $k_{12}$ and $k_{23}$, whereas

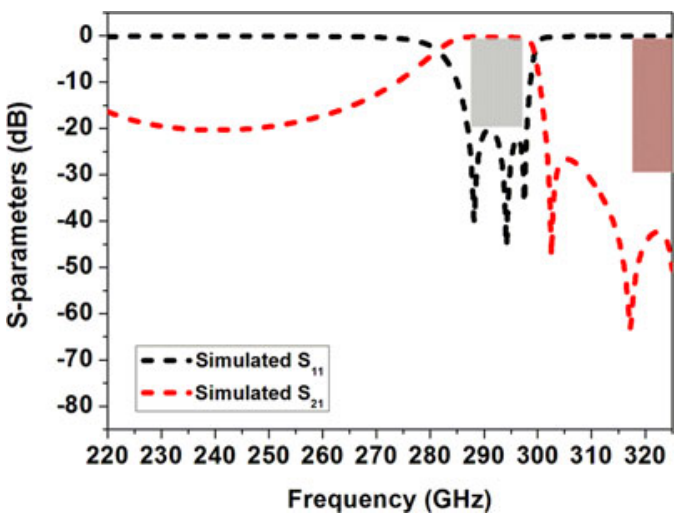

Fig. 4. Simulated $S$-parameters for the SU-8 micromachined filter. Passband and stopband specifications are shown in gray and red, respectively.

the offset $d$ between the test port and first/third resonators controls the external coupling. The cross coupling between first and third resonators is accomplished by a slot in the middle layer. The frequency of the resulting transmission zero is controlled by the width of this cross-coupling slot, $h_{c}$. The above geometric design parameters, shown in Fig. 3, are optimized by the SMEAFO method [23] using CST Microwave Studio (version 2016). Fig. 3 provides the detailed dimensions of this filter after optimization.

The filter is designed to be inserted between standard UG387 waveguide flanges for measurement. As shown in Fig. 3(b), holes to accommodate the flange dowels, thereby accurately aligning the different SU-8 layers, are incorporated in the design. Larger clearance holes for the waveguide flange screws are also introduced.

The optimized $S$-parameter response of the filter is shown in Fig. 4. The predicted passband insertion loss is below $0.4 \mathrm{~dB}$ and the rejection in the unwanted sideband is better than $35 \mathrm{~dB}$. Passband reflectivity, $S_{11}$, is below $-20 \mathrm{~dB}$. The simulations predict two unexpected transmission zeros, one located between passband and upper stopband at $302.5 \mathrm{GHz}$ and another at $326.2 \mathrm{GHz}$, just above the waveguide band's upper edge. These zeros are caused by unwanted cancellation effect of the signals transmitted by different paths from the input to the output due to the special structure of the design. That is, apart from cross coupling accomplished by the slot on the middle layer, there is an additional coupling path between resonators 1 and 3 .

\section{FABRICATION DETAILS}

The extracted pole waveguide filter was fabricated at the Rutherford Appleton Laboratory using a high-precision Kern milling machine and tungsten carbide cutters with diameters down to $0.20 \mathrm{~mm}$. The block material was copper alloy which was then coated with a thin film of gold (around $3 \mu \mathrm{m}$ ) by electroplating. As mentioned above, the internal corners of the filter had a radius of $0.2 \mathrm{~mm}$. Fig. 5 shows a photograph of the split plane of the $\mathrm{CNC}$ machined block with its four resonators.

The fabricated layers of the SU-8 filter are shown in Fig. 6(a). The process details for the SU-8 filter can be found in [13], [24], 


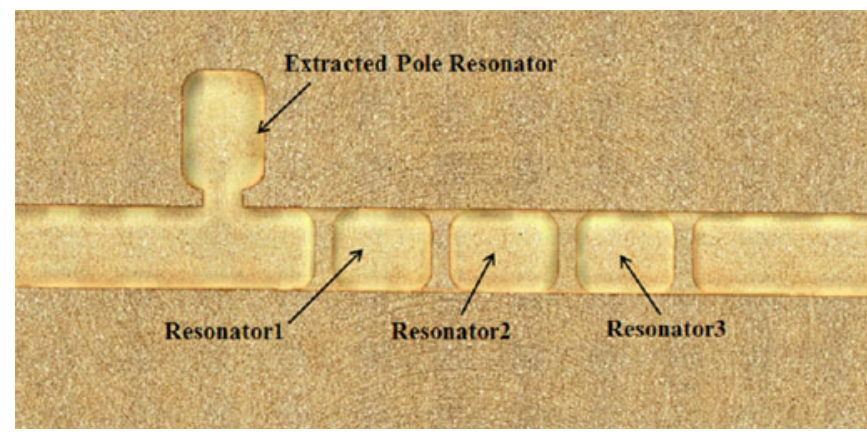

Fig. 5. Photograph of the internal cavities of the fabricated CNC milled extracted pole filter.

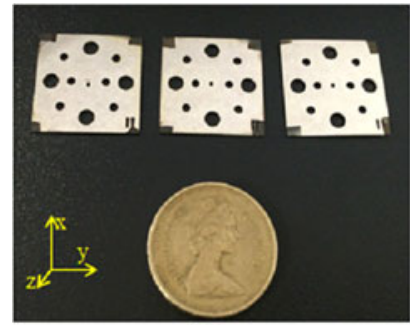

(a)

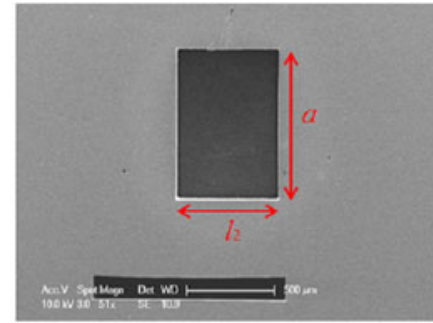

(b)
Fig. 6. (a) Photograph of the three silver-coated SU-8 layers, $20 \times 20 \mathrm{~mm}$ for each layer with a thickness of $0.432 \mathrm{~mm}$. (b) Scanning electron microscope image of the cavity structure for the second SU-8 layer.

and [25]. In summary, a single-side-polished 100-mm-diameter 1-mm-thick silicon wafer was used as a base for forming the SU-8. OmniCoat from MicroChem was used as a 1.5- $\mu \mathrm{m}$-thick sacrificial layer between SU-8 and silicon. This thickness of OmniCoat was built up from five 300-nm-thick layers. Each was formed from precursor spun at $350 \mathrm{r} / \mathrm{min}$ for $10 \mathrm{~s}$ and then at $700 \mathrm{r} / \mathrm{min}$ for $20 \mathrm{~s}$. The coating was soft baked by placing the wafer on a hot plate at $200{ }^{\circ} \mathrm{C}$ for $120 \mathrm{~s}$. It was then cooled on a flat copper plate at room temperature for $300 \mathrm{~s}$.

SU-8 50 negative photoresist from MicroChem was then spin coated onto the OmniCoat. The thickness of the SU-8 layer is calculated by measuring the mass of the coating [13]. A mass of $5.20 \mathrm{~g}$ on a $100-\mathrm{mm}$-diameter wafer corresponds to a thickness of $432 \mu \mathrm{m}$. The coated wafer is left on a leveled copper plate at room temperature for $1 \mathrm{~h}$ for self-planarization. Then it was soft baked at 65 and $85{ }^{\circ} \mathrm{C}$ for 40 and $240 \mathrm{~min}$, respectively. In order to reduce the thermal stresses during soft bake, the temperature was increased at $5{ }^{\circ} \mathrm{C} / \mathrm{min}$ from room temperature during heating. During cooling, the hotplate and wafer cooled naturally to room temperature after switching off the power. UV photolithography was carried out in Cannon PLA-510 mask aligner. The resist was exposed for 4 cycles of $40 \mathrm{~s}$, with a 2-min interval between each cycle to allow the resist to stabilize. A PL 360 filter was placed over the chrome mask during UV exposure, which effectively blocked UV radiation with a wavelength below the $365-\mathrm{nm}$ i-line [26]. The exposed wafers were baked at $70{ }^{\circ} \mathrm{C}$ for $30 \mathrm{~min}$, which helps the acid-assisted cross linking of the exposed structures. After this bake, the SU-8/Si wafer was developed for $15 \mathrm{~min}$ in MicroChem EC at room temperature with constant magnetic

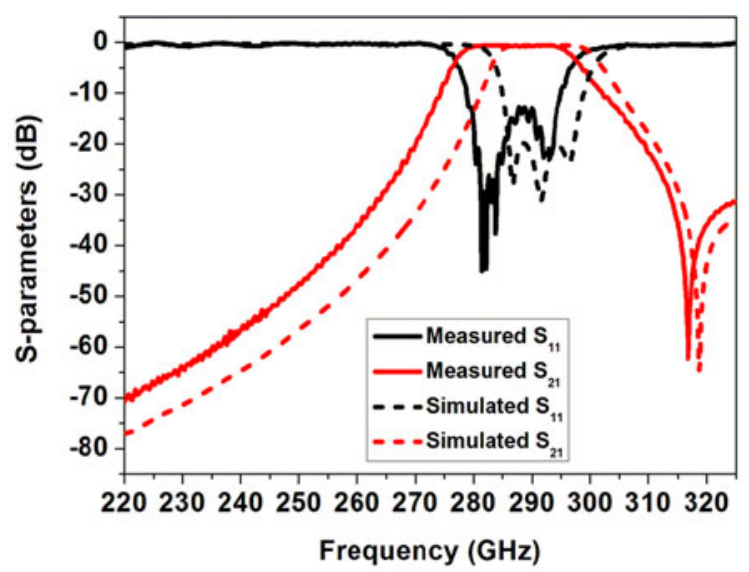

(a)

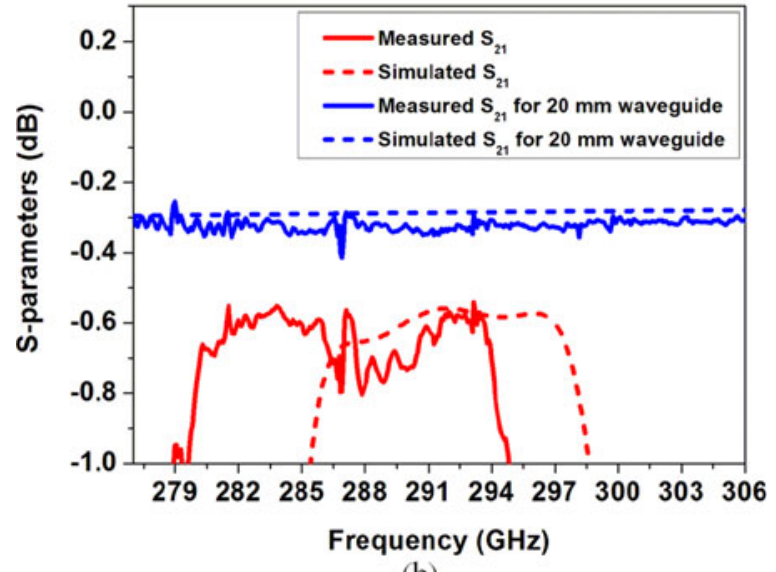

(b)

Fig. 7. Comparison of the simulated and measured results of the filter produced by CNC milling. (a) Response over whole WR-3 band. (b) Expanded view of $S_{21}$ showing the passband. The simulations are performed assuming a material conductivity corresponding to that of bulk gold.

stirring. The patterned SU-8 layers were released by dissolving the sacrificial layer in tetramethylammonium hydroxide-based MFCD26 solution from MicroChem at room temperature for $5 \mathrm{~h}$. The released SU-8 pieces were cleaned with propan-2-ol and dried by nitrogen gas. Layers of $30 \mathrm{~nm}$ of chromium, and subsequently $1500 \mathrm{~nm}$ of silver, were deposited by sputtering and thermal evaporation, respectively. This was done on both sides of the patterned SU-8 layers without breaking the vacuum. A purpose-built sample-tilted-rotating rig allowed the metal to reach the inner walls of the waveguides and cavities.

\section{MEASUREMENT AND DISCUSSION}

The $S$-parameter measurements for the CNC milled extracted pole filter were carried out using a Keysight PNA network analyzer with a pair of VDI (Virginia Diodes, Inc.) WR-3.4 extension heads. For the measurement, the $\mathrm{CNC}$ machined block was fixed between the waveguide flanges of two frequency extension heads. The insertion loss for the 20-mm length of WR-3 waveguide in the same block was also measured. The results are shown in Fig. 7. The filter has an average passband insertion loss of around $0.65 \mathrm{~dB}$ and greater than $30-\mathrm{dB}$ rejection in the upper stopband. As shown in Fig. 7(b), the insertion loss for 
TABLE I

COMParison BETWEen DESIGNED AND MEASUREd RESONATOR DiMENSIONS FOR THE CNC MILLED EXTRACTED POLE FILTER

\begin{tabular}{lcc}
\hline \hline & Designed $(\mu \mathrm{m})$ & Measured $(\mu \mathrm{m})$ \\
\hline Cavity \# & $a \times b \times l$ & $a \times b \times l$ \\
Resonator 1 & $864 \times 432 \times 510$ & $874 \times 438 \times 521$ \\
Resonator 2 & $864 \times 432 \times 567$ & $874 \times 438 \times 574$ \\
Resonator 3 & $864 \times 432 \times 510$ & $874 \times 438 \times 519$ \\
\hline \hline
\end{tabular}

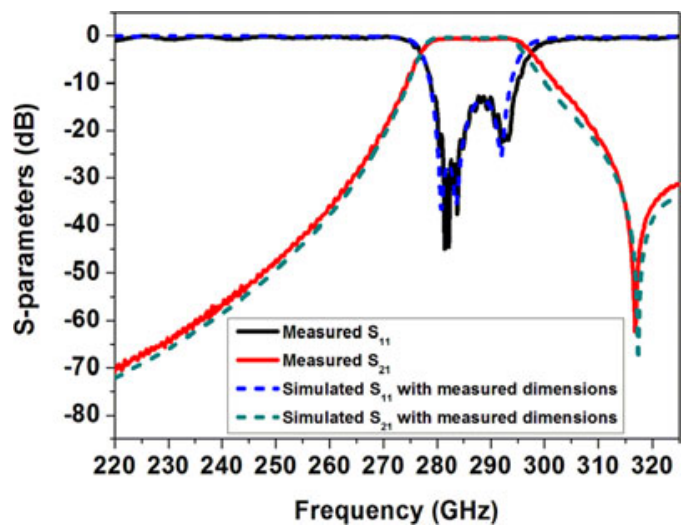

Fig. 8. Simulated response of the CNC milled extracted pole filter with measured dimensions shown in Table I.

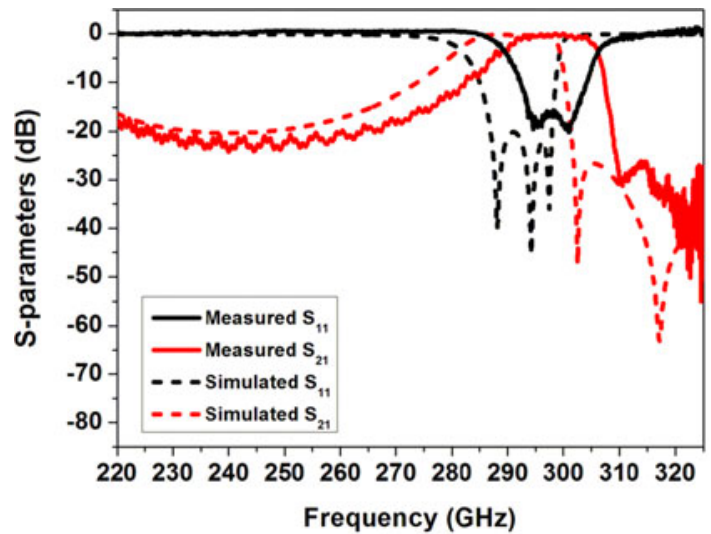

(a)

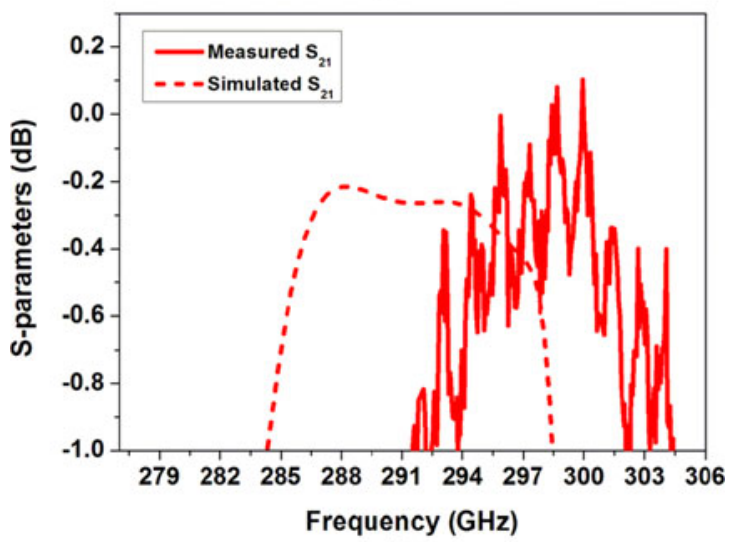

(b)

Fig. 9. Comparison of the simulated results and measured results of the filter based on SU-8 photoresist technology. (a) Response over whole WR-3 band. (b) Expanded view of $S_{21}$ over passband. The simulations are performed in CST assuming a material conductivity equal to that of silver.
TABLE II

COMPARISON BETWEEN DESIGNED AND MEASUREd RESONATOR DimENSIONS FOR THE SU-8 FILTER

\begin{tabular}{lccc}
\hline \hline & \multirow{2}{*}{ Designed $(\mu \mathrm{m})$} & \multicolumn{2}{c}{ Measured $(\mu \mathrm{m})$} \\
\cline { 3 - 4 } & & Side 1 & Side 2 \\
\hline Cavity \# & $a \times l$ & $a \times l$ & $a \times l$ \\
Resonator 1 & $876 \times 647$ & $863 \times 623$ & $869 \times 638$ \\
Resonator 2 & $876 \times 589$ & $861 \times 576$ & $874 \times 584$ \\
Resonator 3 & $876 \times 647$ & $855 \times 630$ & $873 \times 641$ \\
\hline \hline
\end{tabular}

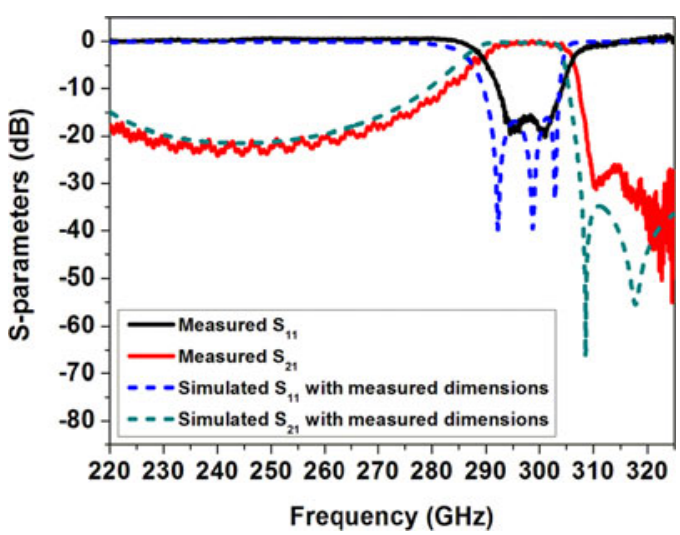

Fig. 10. Simulated response of the SU-8 filter with measured dimensions in Table II.

TABLE III

Comparison of Measurements Between CNC Milled Extracted Pole FILTER AND SU-8 MiCROMACHINED FILTER

\begin{tabular}{lcc}
\hline \hline & $\begin{array}{c}\text { CNC extracted } \\
\text { pole filter }\end{array}$ & SU-8 filter \\
\hline Passband insertion loss & $0.41 \mathrm{~dB}$ & $0.45 \mathrm{~dB}$ \\
Passband frequency shift & $-5 \mathrm{GHz}$ & $7 \mathrm{GHz}$ \\
Stopband attenuation & $>32 \mathrm{~dB}$ & $>33 \mathrm{~dB}$ \\
Size of filter device & $\begin{array}{c}20 \mathrm{~mm} \times 20 \mathrm{~mm} \\
\times 4 \mathrm{~mm}\end{array}$ & $\begin{array}{c}19 \mathrm{~mm} \times 19 \mathrm{~mm} \times \\
\end{array}$ \\
\hline
\end{tabular}

$20 \mathrm{~mm}$ of waveguide is measured to be $0.3 \mathrm{~dB}$, giving the waveguide a loss of $0.015 \mathrm{~dB} / \mathrm{mm}$. Allowing for a total of 16 $\mathrm{mm}$ of waveguide connecting the filter to the flanges, the loss of the 4-mm-long filter structure is thus $0.41 \mathrm{~dB}$.

The measurements are in very good agreement with simulations except that center frequency of the filter is shifted downwards by around $5 \mathrm{GHz}$. This frequency shift is mainly due to larger-than-designed dimensions of resonators, which have been measured with results shown in Table I. Generally dimensions are within a few micrometers of designed values, except for the width of the waveguide which is about $15 \mu \mathrm{m}$ larger than assumed in the simulations. When the filter is resimulated with CST using the measured dimensions, excellent agreement with measurements is obtained as shown in Fig. 8.

For the SU-8 filter, the measurement is performed on an Agilent E8361A network analyzer using a short-open-load-thru calibration. The SU-8 filter is placed between two waveguide flanges of the network analyzer and the layers aligned by the high precision dowels on the waveguide flanges. 
TABLE IV

Comparison of Recently Published Waveguide BandPass Filter OPERATING IN Frequency Range From WR-3 To WR-1.5 Band

\begin{tabular}{|c|c|c|c|c|c|c|c|c|}
\hline $\begin{array}{l}\text { Waveguide } \\
\text { band }\end{array}$ & $f_{0}(\mathrm{GHz})$ & $F B W$ & $\begin{array}{l}\text { Micromachining } \\
\text { techniques }\end{array}$ & Filter response & $n$ & $I L(\mathrm{~dB})$ & $R L(\mathrm{~dB})$ & Reference (year) \\
\hline WR-3 & 257.7 & $8.77 \%$ & $\mathrm{CNC}$ ( $H$-plane split) & $\begin{array}{l}\text { Quasi-elliptical, one TZ at lower stopband } \\
\text { and one upper stopband }\end{array}$ & 4 & 0.7 & $>14$ & [6] (2017) \\
\hline WR-3 & 256.3 & $9.83 \%$ & $\mathrm{CNC}$ ( $H$-plane split) & $\begin{array}{l}\text { Quasi-elliptical, one TZ at lower stopband } \\
\text { and one upper stopband }\end{array}$ & 4 & 0.5 & $>15$ & [6] (2017) \\
\hline WR-3 & 309.35 & $4.4 \%$ & SU-8 (three layers) & Chebyshev, one TZ at lower stopband & 3 & 0.4 & $>15$ & [12] (2013) \\
\hline WR-3 & 286.6 & $5.58 \%$ & $\mathrm{CNC}$ (E-plane split) & Chebyshev, one $\mathrm{TZ}$ at upper stopband & 3 & 0.41 & $>14$ & This work \\
\hline WR-1.5 & 671 & $7.91 \%$ & SU-8 (three layers) & Chebyshev, one $\mathrm{TZ}$ at lower stopband & 3 & 0.65 & $>11$ & [13] (2013) \\
\hline
\end{tabular}

Note: $f_{0}$ : center frequency of the filter; $F B W$ : fractional bandwidth; TZ: transmission zero; $n$ : filter order; $I L$ : passband insertion loss; $R L$ : passband return loss.

The measured results for the SU-8 filter are shown in Fig. 9. It can be observed from Fig. 9(b) that there are significant ripples in the measured $S_{21}$ response. A different network analyzer was used in these measurements to those of the CNC filter and the ripple is attributed to a poor match in the receiver head which has a transmit-only module at Port 2 . This problem is discussed in detail in [27]. The measured insertion loss is around $0.45 \mathrm{~dB}$ in the passband and a larger than $30-\mathrm{dB}$ rejection within the upper stopband. However, the center frequency of this filter is shifted upward by around $7 \mathrm{GHz}$ from the simulation. Because the layer thickness determines the resonator height $b$, simulations show that the center frequency of the filter does not strongly depend on the layer thickness. So, the difference in center frequency of the filter between measurement and simulation may be due to inaccurate dimensions in the layer plane. The dimensions of the SU-8 filter cavities were measured using a scanning electron microscope. The image in Fig. 6(b) shows the cavity within the second layer and Table II shows the measured resonator dimensions. Measured dimensions in the plane are around $1 \%$ smaller than designed values on one side of the SU-8 layer and 3\% smaller on the other side, which means that the frequency shift may be caused by both changed dimensions and nonvertical sidewalls. After inserting the measured dimensions in CST simulations, a much improved agreement with measurements is obtained, as shown in Fig. 10.

Table III shows a comparison of measurements on the CNC extracted pole filer and the SU-8 micromachined filter. Both filters comfortably achieve the requirements of a lower than 0.6-dB passband insertion loss, with measured values of 0.41 and 0.45 $\mathrm{dB}$ for the metal and SU-8 devices, respectively. The requirements for over 30-dB stopband attenuation are also achieved. The CNC milled extracted pole filter offers a better low frequency rejection, whereas the SU-8 device provides a steeper roll-off on this high frequency side of the passband and a smaller overall volume.

Table IV shows the comparison between waveguide filters reported in open literature and two filters described here. These filters operate in the frequency range from WR-3 band to WR-1.5 band. A comparison of some WR-10 band waveguide filters can be found in [14]. All of the filters summarized in Table IV are based on coupled rectangular resonant cavities, but fabricated using different micromachining techniques. Care must be taken with the comparisons as the filters have different specifications.

\section{CONCLUSION}

A CNC milled extracted pole waveguide filter and a SU-8 micromachined filter working in WR-3 band have been designed to best exploit the characteristics of the fabrication processes. It is the first demonstration that a CNC milled filter with a steep rejection characteristic beyond $300 \mathrm{GHz}$ and a SU-8 micromachined filter with novel cross-coupling topology working at WR-3 band. The measured performance of the filters is in very good agreement with the numerical predictions once the latter have been corrected for the small errors in manufacturing. The two filters show no significant difference in performance despite the two alternative fabrication processes and designs. Both filters achieve an insertion loss of below $0.5 \mathrm{~dB}$ in a $\sim 10$-GHz-wide passband and a rejection of more than $30 \mathrm{~dB}$ in the stopband. Either of these two micromachined waveguide filters can therefore be potential replacements of FSS filters used in spaceborne radiometers for unwanted sideband rejection.

\section{REFERENCES}

[1] R. J. Martin and D. H. Martin, "Quasi-optical antennas for radiometric remote sensing," Electron. Commun. Eng. J., vol. 8, pp. 37-48, Feb. 1996.

[2] R. Cahill et al., "Low loss FSS for channel demultiplexing and image band rejection filtering," in Proc. 24th ESTEC Antenna Workshop Innov. Periodic Antennas Photon. Bandgap Fractal Freq. Sel. Surf., The Netherlands, May 2001, pp. 103-108, European Space Agency.

[3] R. Dickie et al., "Submillimeter wave frequency selective surface with polarization independent spectral responses," IEEE Trans. Antennas Propag., vol. 57, no. 7, pp. 1985-1994, Jul. 2009

[4] X. Liao, L. Wan, Y. Yin, and Y. Zhang, "W-band low-loss bandpass filter using rectangular resonant cavities," IET Microw. Antennas Propag. vol. 8, no. 15, pp. 1440-1444, Sep. 2014.

[5] C. A. Leal-Sevillano, J. R. Montejo-Garai, J. A. Ruiz-Cruz, and J. M. Rebollar, "Low-loss elliptical response filter at $100 \mathrm{GHz}$," IEEE Microw. Wireless Compon. Lett., vol. 22, no. 9, pp. 459-461, Sep. 2012.

[6] J. Q. Ding et al., "WR-3 band quasi-elliptical waveguide filters using higher order mode resonances," IEEE Trans. THz Sci. Technol., vol. 7, no. 3, pp. 302-309, May 2017.

[7] H. V. Jansen, M. J. de Boer, S. Unnikrishnan, M. C. Louwerse, and M. C. Elwenspoek, "Black silicon method X: A review on high speed and selective plasma etching of silicon with profile control: An in-depth comparison between Bosch and cryostat DRIE processes as a roadmap to next generation equipment," J. Micromech. Microeng., vol. 19, no. 3, pp. 033 001-1-033 001-41, Feb. 2009. 
[8] J. Y. Li, B. Pan, C. Lugo, M. Tentzeris, and J. Papapolymerou, "Design and characterization of a W-band micromachined cavity filter including a novel integrated transition from CPW feeding lines," IEEE Trans. Microw. Theory Techn., vol. 55, no. 12, pp. 2902-2910, Dec. 2007.

[9] K. M. K. H. Leong et al., "WR1.5 silicon micromachined waveguide components and active circuit integration methodology," IEEE Trans. Microw. Theory Techn., vol. 60, no. 4, pp. 998-1005, Apr. 2012.

[10] J. R. Stanec and N. S. Barker, "Fabrication and integration of micromachined submillimeter-wave circuits," IEEE Microw. Wireless Compon. Lett., vol. 21, no. 8, pp. 409-411, Aug. 2011.

[11] X. Shang, M. Ke, Y. Wang, and M. J. Lancaster, "Micromachined W-band waveguide and filter with two embedded H-plane bends," IET Microw. Antennas Propag., vol. 5, no. 3, pp. 334-339, Feb. 2011.

[12] Q. Chen, X. Shang, Y. Tian, J. Xu, and M. J. Lancaster, "SU-8 micromachined WR-3 band waveguide bandpass filter with low insertion loss," Electron Lett., vol. 49, no. 7, pp. 480-482, Mar. 2013.

[13] X. Shang, Y. Tian, M. J. Lancaster, and S. Singh, "A SU8 micromachined WR-1.5 band waveguide filter," IEEE Microw. Wireless Compon. Lett., vol. 23, no. 6, pp. 300-302, Jun. 2013.

[14] X. Shang et al., "W-band waveguide filters fabricated by laser micromachining and 3-D printing," IEEE Trans. Microw. Theory Techn., vol. 64, no. 8, pp. 2572-2580, Aug. 2016.

[15] C. H. Smith, A. Sklavonuos, and N. S. Barker, "SU-8 micromachining of millimeter and submillimeter waveguide circuits," in IEEE MTT-S Int. Microw. Symp. Dig., Boston, MA, USA, 2009, pp. 961-964.

[16] J. Bornemann, "A new class of E-plane integrated millimeter-wave filters," in IEEE MTT-S Int. Microw. Symp. Dig., Long Beach, CA, USA, vol. 2, 1989, pp. 599-602.

[17] J. Hong and M. J. Lancaster, Microstrip Filters for RF/Microwave Applications. New York, NY, USA: Wiley, 2001.

[18] R. R. Mansour and G. Woods, "Design of millimeter-wave extracted-pole filters with asymmetrical frequency characteristics," in IEEE MTT-S Int. Microw. Symp. Dig., Boston, MA, USA, vol. 2, 1991, pp. 659-663.

[19] J. D. Rhodes and R. J. Cameron, "General extracted pole synthesis technique with applications to low-loss $\mathrm{TE}_{011}$ mode filters," IEEE Trans. Microw. Theory Techn., vol. 28, no. 9, pp. 1018-1028, Sep. 1980.

[20] Y. Yang, M. Yu, and Q. Wu, "Advanced synthesis technique for unified extracted pole filters," IEEE Trans. Microw. Theory Techn., vol. 64, no. 12, pp. 4463-4472, Dec. 2016.

[21] J. R. Montejo-Garai et al., "Compact low-cost diplexer with elliptic filter response for Ka-band satellite applications," in Proc. 2015 Int. Workshop Microw. Filters, 2015, pp. 1-3.

[22] C. H. Smith III, H. Xu, and N. S. Barker, "Development of a multilayer SU-8 process for terahertz frequency waveguide blocks," in IEEE MTT-S Int. Microw. Symp. Dig., 2005, pp. 439-442.

[23] B. Liu, H. Yang, and M. J. Lancaster, "Global optimization of microwave filters based on a surrogate model-assisted evolutionary algorithm," IEEE Trans. Microw. Theory Techn., vol. 65, no. 6, pp. 1976-1985, Jun. 2017.

[24] J. D. Williams and W. Wang, "Study on the postbaking process and the effects on UV lithography of high aspect ratio SU-8 microstructures," $J$. Microlithogr. Microfab. Microsyst., vol. 3, pp. 563-568, 2004.

[25] X. Shang, M. Ke, Y. Wang, and M. J. Lancaster, "WR-3 band waveguides and filters fabricated using SU8 photoresist micromachining technology," IEEE Trans. THz Sci. Technol., vol. 2, no. 6, pp. 629-637, Oct. 2012.

[26] D. Glynn et al., "Submillimetre rectangular waveguides based on SU-8 photoresist micromachining technology," in Proc. 2016 46th Eur. Microw. Conf., London, U.K., 2016, pp. 1346-1349.

[27] Y. Wang, M. J. Lancaster, M. Ke, and X. Shang, "Measurements of micromachined waveguide devices at WR-3 band using a T/R-T module based network analyzer," in Proc. 77th ARFTG Microw. Meas. Conf., Baltimore, MD, USA, 2011, pp. 1-4.

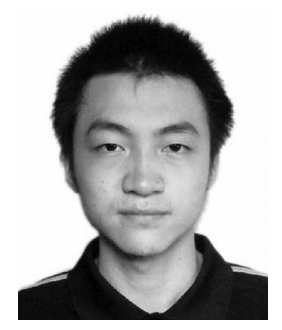

Hao Yang was born in Wuhan, China, in 1991. He received the B.Eng. degree in electronics and information engineering from the Huazhong University of Science and Technology, Wuhan, in 2014, and the B.Eng. degree in electronic and electrical engineering from the University of Birmingham, Birmingham, U.K., in 2014, where he is currently working toward the Ph.D. degree.

His current research interests include terahertz frequency filters and mutiplexers.

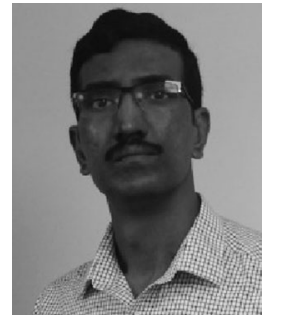

Yuvaraj Dhayalan received the M.Sc. degree in materials science from the College of Engineering, Anna University, Chennai, India, in 2004, and the Ph.D. degree from the Indian Institute of Science, Bangalore, India, in 2010.

He has been with the University of Birmingham, Birmingham, U.K., as a Research Fellow since 2015. His research interests are in thin film, nanoscale devices, microfabrication, and nanofabrication.

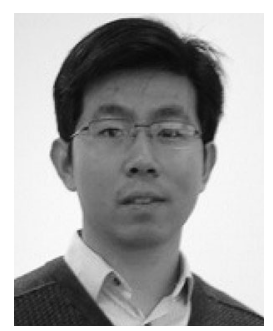

Xiaobang Shang (M'13) was born in Hubei, China, in 1986. He received the B.Eng. degree (first class) in electronic and communication engineering from the University of Birmingham, Birmingham, U.K., in 2008, the B.Eng. degree in electronics and information engineering from Huazhong University of Science and Technology, Wuhan, China, in 2008, and the $\mathrm{Ph} . \mathrm{D}$. degree in microwave engineering from the University of Birmingham, in 2011. His doctoral research concerned micromachined terahertz waveguide circuits and synthesis of multiband filters.

He is currently a Senior Research Scientist with the National Physical Laboratory (NPL), U.K. Prior to joining the NPL, he was a Research Fellow with the University of Birmingham. His current main research interests include microwave measurements, microwave filters and multiplexers, and micromachining techniques.

Dr. Shang was the recipient of the ARFTG Microwave Measurement Student Fellowship Award in 2009 and the co-recipient of the Tatsuo Itoh Award in 2017.

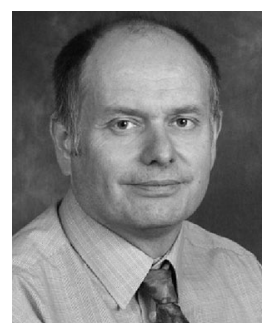

Michael J. Lancaster (SM'04) was born in U.K., in 1958. He received the Graduation degree in physics and Ph.D. dergree for research into nonlinear underwater acoustics from Bath University, Bath, U.K., in 1980 and 1984, respectively.

After leaving Bath University, he joined the Surface Acoustic Wave (SAW) Group, Department of Engineering Science, Oxford University, as a Research Fellow. His research was in the design of new, novel SAW devices, including RF filters and filter banks. In 1987, he became a Lecturer with the Department of Electronic and Electrical Engineering, University of Birmingham, Birmingham, U.K., lecturing in electromagnetic theory and microwave engineering. Shortly after he joined the department, he began the study of the science and applications of high-temperature superconductors, working mainly at microwave frequencies. He was promoted to the Head of the Department of Electronic, Electrical and Systems Engineering in 2003. He has authored or coauthored 2 books and more than 200 papers in refereed journals. His research interests include microwave filters and antennas, as well as the high frequency properties and applications of a number of novel and diverse materials. This includes micromachining as applied to terahertz communications devices and systems.

Dr. Lancaster is a Fellow of the Institution of Engineering and Technology and the U.K. Institute of Physics. He is a Chartered Engineer and a Chartered Physicist. He has served on the IEEE Microwaves and Theory and Techniques Society International Microwave Symposium Technical Committees.

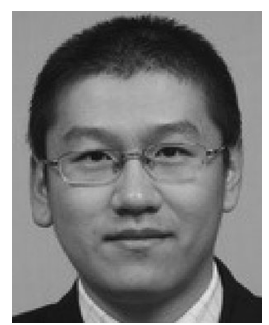

Bo Liu (M'15-SM'17) received the B.S. degree from Tsinghua University, Beijing, China, in 2008, and the $\mathrm{Ph} . \mathrm{D}$. degree from the MICAS Laboratories, University of Leuven (KU Leuven), Leuven, Belgium, in 2012.

From 2012 to 2013, he was a Humboldt Research Fellow and was with the Technical University of Dortmund, Dorthmund, Germany. In 2013, he was appointed a Lecturer with Wrexham Glyndwr University, Wrexham, U.K., where he was promoted to a Reader in computer-aided design in 2016. He has authored or coauthored 1 book and more than 40 papers in international journals, edited books, and conference proceedings. His research interests include design automation methodologies of analog/RF integrated circuits, microwave devices, microelectromechanical systems, evolutionary computation and machine learning.

Dr. Liu is an Honorary Fellow of the University of Birmingham, Birmingham, U.K. 


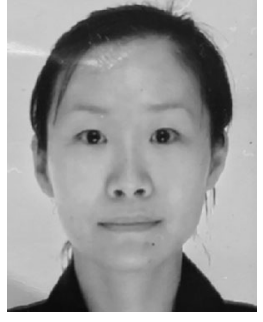

Hui Wang received the M.Sc. and Ph.D. degrees in astrophysics and space instrumentation from the University Pierre and Marie Curie, Paris, France, in 2005 and 2009, respectively.

She joined the Millimetre Wave Technology Group, STFC Rutherford Appleton Laboratory, Oxfordshire, U.K., in 2009, where she is currently leading mixer device development within the group. Her current research interests include millimeterwave and terahertz devices, primarily heterodyne frequency mixers and harmonic up-conversion multipliers, in support of earth observation and astronomy remote sounding experiments.

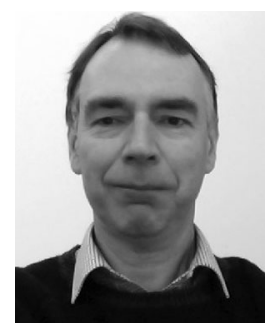

Peter G. Huggard (M'11-SM'12) received the B.A. (Mod) degree in experimental physics and Ph.D. degree from the University of Dublin, Trinity College, Dublin, Ireland, in 1986 and 1991, respectively.

Since 2000, he has been a member of the Millimetre Wave Technology Group, UK's STFC Rutherford Appleton Laboratory, Oxfordshire, U.K. His current research interests include developing filters, sources and detectors for gigahertz and terahertz radiation.

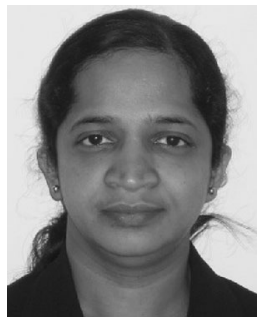

Manju Henry received the Master's and Ph.D. degrees in electronic engineering from the Cochin University of Science and Technology, Kerala, India, in 1998 and 2002, respectively.

After receiving the $\mathrm{Ph}$.D. degree, she had done five years of postdoctoral studies with the Institute of High Frequency and Microwave Techniques, Karlsruhe Institute of Technology, the former FZK, Karlsruhe, Germany, and at the University of Surrey, Guildford, U.K. She joined the Millimetre Wave Technology Group, STFC Rutherford Appleton Laboratory, Oxfordshire, U.K., in 2007. After joining the group, she had undertaken key technical and management roles in several European Union/European Space Agency programs. She is currently involved in a wide range of tasks that include millimeter-wave passive system design for atmospheric sounding and astronomy, active system development for meteorological remote sounding, and security imaging. 